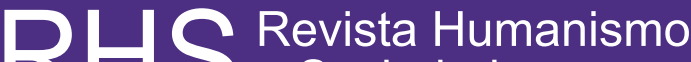

RHsin=

\section{Teología de la \\ Liberación: ¿aporte epistemológico ausente en la enseñanza de las ciencias sociales hoy?}

\begin{abstract}
Andrés Esteban Cardona González
\end{abstract}
andrescardona.0319@gmail.com

https://doi.org/10.22209/rhs.v6n1a05

Recibido: diciembre 6 de 2017.

Aceptado: abril 16 de 2018.

\section{Resumen}

La enseñanza de las ciencias sociales hoy se ve permeada por el discurso positivista de las ciencias imposibilitando el desarrollo de conocimientos desde el contexto. Este artículo pretende argumentar que la epistemología de la Teología de la Liberación, como experiencia liberadora y crítica, permite que se inicie con la construcción de la epistemología tanto de la libertad como de la realidad histórica y de un discurso latinoamericano a partir de las instituciones educativas en medio de la labor del docente de ciencias sociales. El proceso investigativo ha sido indagado desde la fenomenología hermenéutica y entrega una manera de enseñar ciencias sociales que debería tenerse en cuenta si se pretende la construcción del pensamiento crítico del estudiante.

Palabras Clave: epistemología latinoamericana, currículo, pedagogía, didáctica, maestro, Teología de la Liberación.
Liberation theology: an epistemological contribution lacking in the teaching of social sciences today?

\section{Abstract}

The teaching of social sciences today is permeated by the positivist discourse of sciences, which makes it impossible to develop knowledge from the context. This article argues that liberation theology epistemology, as a liberating and critical experience, enables the development of the epistemology of freedom and historical reality, as well as of a Latin American discourse based on educational institutions where social science teachers perform their jobs. The research process is addressed using the hermeneutical phenomenology approach and a way to teach social sciences is proposed that should be taken into account if the aim is to develop critical thinking skills among students.

Keywords: Latin American epistemology, curriculum, pedagogy, didactics, teacher, Liberation Theology.

1) Profesor de Ciencias Sociales del Colegio Nuestra Señora del Rosario de Chiquinquirá, Medellín. 


\section{Introducción}

Para los años 60, en Suramérica se dan las primeras iniciativas metodológicas y las bases epistemológicas en la enseñanza de las ciencias sociales; en este orden de ideas, surge la Investigación Acción Participativa (IAP) propuesta por Orlando Fals Borda y Camilo Torres, con la que se logró una incidencia científica propia en el contexto latinoamericano, simultáneamente el fortalecimiento de los movimientos sociales posibilitó el desarrollo de dichas iniciativas.

La Teología de la Liberación, ${ }^{2}$ desde de las denominadas Comunidades Eclesiales de Base, surge para contraponerse al discurso metodológico europeo. Este movimiento propone como base un método, que si bien en aquella época se implementa como estrategia de evangelización de carácter emancipador como el llamado método «Ver, Juzgar y Actuar», será el pilar para la construcción de la IAP y del surgimiento de epistemologías como: la Pedagogía de la Liberación de Paulo Freire, quien fue influido en gran parte por la Teología de la Liberación y su método; la Ética y la Filosofía de la Liberación de Enrique Dussel; la Educación Popular, entre otras tantas, que fueron vislumbrando en el marco de América Latina durante la segunda mitad del Siglo XX y que hasta hoy vienen abriéndose espacio en los discursos globales.

«Teología de la Liberación: ¿aporte epistemológico ausente en la enseñanza de las ciencias

2| En adelante $T L$, es un movimiento social teológico creado por religiosos de América a mediados de la década de 1960, fundado por Gustavo Gutiérrez (Perú), Leonardo Boff (Brasil) y Camilo Torres (Colombia); oficializado en la Conferencia Episcopal Latinoamericana (CELAM) de Medellín en 1968. sociales hoy?» busca mostrar al lector que, en un mundo cada vez más complejo, cambiante y desafiante, es apremiante que las personas se nutran de conocimiento y herramientas provenientes de las ciencias sociales para que comprendan su realidad (MEN, 2003, p. 104), se apropien de ella y recreen las ciencias sociales y su enseñanza desde las epistemologías latinoamericanas más allá de la transversalización del currículo en línea positivista ${ }^{3}$ El artículo inicialmente desarrolla la epistemología de la Teología de la Liberación, para establecer una conexión de sus fundamentos con lo que podría aportar a la enseñanza de las ciencias sociales desde la construcción del discurso latinoamericano y, finalmente, plantea una discusión sobre el papel del docente en la enseñanza de las ciencias sociales en la construcción del discurso desde Latinoamérica.

\section{Metodología}

Este artículo tiene como antecedente una investigación amplia realizada en el semillero «Contexto Pedagógico» de la Universidad Autónoma Latinoamericana, por medio de la cual se hizo una lectura rigurosa de textos base de TL, en la que son explicadas características tales como su esencia, su arché desde la fe, su método y sus posibles impactos en el desarrollo de los sujetos pensantes que lleven su carácter de cristianos a una coherencia con su praxis.

3| Si se revisan los lineamientos curriculares de ciencias sociales, puede observarse que solo aparece para Latinoamérica algo asociado a «latinoamericanismos». Pero no se hace explícita la existencia de un discurso latinoamericano. Se recomienda al lector hacer el ejercicio de buscar la palabra 'Latinoamérica' dentro de dichos lineamientos. 
Desde lo planteado por Tomás Domingo Moratalla (2001) en «La fenomenología hermenéutica de Paul Ricoeur: mundo de la vida e imaginación», se parte y se hace hincapié en que para este autor la imaginación abre horizontes que son de vital importancia para conectarse con otras comunidades y culturas. Esa imaginación genera empatía para salir del globo de nosotros mismos, es decir, lograr la capacidad de interpretar la realidad del otro sin tener como prejuicio mi individualidad para identificarme con él. A partir de lo anterior, se implementaron las fases metodológicas sin perder de vista que el yo sea un sí, pero pasando por el otro, poniéndose en la piel del otro (Domingo Moratalla, p. 301), en consonancia con una hermenéutica interpretativa que abra puertas a la construcción de conocimientos propios de Latinoamérica, como un aporte a la enseñanza de las ciencias sociales

Por lo tanto, surgen tres fases: descriptiva, integrativa y propositiva, con las que se pretendió una articulación entre el pensamiento alterno de la TL con el carácter positivista que hasta hoy predomina en la enseñanza de las ciencias sociales, tal y como lo orienta el Ministerio de Educación Nacional (MEN).

\section{Fase descriptiva: bases teóricas de la Teología de la Liberación y su epistemología}

La TL se entiende como «el momento teológico de la experiencia realizada por la fe cristiana al resumir conscientemente, desde el evangelio, la transformación del mundo en situación de dependencia» (Boff, 1975, p. 49). Esta surge en un momento histórico (1966) en el que América Latina atravesaba una situación sociopolítica, marcada por gobiernos dictatoriales promovidos por Estados Unidos a través de la
Operación Cóndor, también llamada doctrina Reagan; con la cual se pretendió imponer las políticas estadounidenses en el Cono Sur como estrategia frente al comunismo, en el marco de lo que se conoce como Guerra Fría.

Metodológicamente, la TL asume la «lectura socio-analítico-estructural de la realidad» (Boff, 1975, p. 46), es decir, que el abordaje se hace desde la crítica a aquellas teorías que ponen en situación de injusticia a América Latina, verbigracia: «el desarrollo capitalista, para mantenerse vigente en la historia necesita un coeficiente elevado de injusticia social» y en esa injusticia era en la que se recaía en el continente (Boff, 1975).

Así mismo, la TL abre la posibilidad de actuar en términos de libertad, suponiendo un giro hermenéutico y la adquisición de un nuevo estado de conciencia ${ }^{4}$ en factores como el político, el económico, el religioso, el de la crítica ideológica, para que así, en una perspectiva de liberación, los contenidos de estos factores puedan adquirir un nuevo sentido (Boff, 1975). Lo anterior permite inferir que la TL está inscrita en el método hermenéutico que ahonda en cada dimensión del ser humano, además, genera una conexión con aquella proyección de praxis consecuente en un hacer y un pensar a favor de los que buscan equidad.

Después de tantos años, en Latinoamérica las brechas que la TL buscaba disminuir, como argumenta Boff citando a Gustavo Gutiérrez, aún no se cierran y el continente continúa siendo explotado a favor de las «potencias» del mundo, en

4| Ese estado de conciencia incluye también una conciencia de clase que será tratada en la discusión y la cual fue implementada por Camilo Torreps para la creación de la Plataforma del Frente Unido del Pueblo. Véase: «Unidad en la diversidad: Camilo Torres y el frente unido del pueblo (aportes para el debate)». 
especial aquellas que en la Operación Cóndor buscaban que su política neoliberal primara. A propósito de una nueva conciencia histórica y de una categoría de liberación, Boff (1975, p. 24) cita:

Uno de los mayores teóricos de la liberación a nivel teológico, Gustavo Merino Gutiérrez, lo ha percibido muy bien: «Procurar la liberación del subcontinente va más allá de la superación de dependencia económica, social y política. Es, más profundamente, ver el devenir de la humanidad como un proceso de emancipación del hombre a lo largo de la historia, orientado hacia una sociedad cualitativamente diferente, en la que el hombre se vea libre a toda servidumbre, en la que sea artífice de su propio destino. Es buscar la construcción de un hombre nuevo[...] Esto es lo que en última instancia sostiene el esfuerzo de liberación en que está empeñado el hombre latinoamericano".

No obstante, para que esta liberación y esa nueva conciencia histórica se genere la TL toma algo denominado «Los signos de los tiempos» según el Concilio Vaticano II, texto emitido desde el Vaticano en 1966 que impulsó un cambio de fondo en medio de la religión católica. Y siendo la TL un movimiento que aparece en el cristianismo latinoamericano, esta se propone partir de las culturas propias del continente, propiciando un análisis de la realidad que no tome como referencia únicamente las ciencias sociales, ya que esta no es la única que se preocupa por lo anteriormente mencionado. Se entiende según esta fase que «en ese análisis de la realidad no actúan solamente preocupaciones nacidas en las ciencias sociales» (Boff, 1975, p. 47), sino también otras de orden histórico-cultural y de cultura popular. En concordancia con lo anterior, las mayorías que se vieron aplastadas comenzaron a crear una cultura del silencio dándole otro sentido a su vida para buscar liberarse aún en medio de la opresión que el contexto les imponía. «En esta línea es donde se van estudiando, en casi todo el continente, la cultura y la religiosidad popular como sementera de valores no afectados por la ideología imperialista y como dinamismo para un auténtico proceso de liberación» (Boff, 1975, p. 47).

A partir de los signos de los tiempos y el análisis de la realidad de la $T L$, Juan Carlos Scanonne escribe El método de la Teología de la Liberación, donde plantea que los seres humanos, desde su religiosidad y espiritualidad, al ser «cristianos iluminados» observan los ensayos teológicos a la luz de estos signos, de la opción por los pobres y de las necesidades del pueblo. Más allá de ello, Scannone (1982) argumenta que la TL contiene en sí tres niveles epistemológicos, los cuales se esbozan a continuación y dan sentido a esta primera fase:

- El primero es «el de las ciencias históricas y sociales», el cual analiza una situación concreta y genera una explicación de sus causas. Estas situaciones son generadas desde el desenvolvimiento del sujeto alrededor de su diario vivir, construyendo desde sí un universo que le permite llevar un proceso de interpretación de la realidad según las vivencias reconocidas en el contexto que le rodea.

- El segundo habla de lo «filosófico», es decir, esa interpretación completa del hombre y su libertad dentro de la historia (autonomía). Encontrando que los sujetos se piensan para asumir las consecuencias de las decisiones tomadas sin importar el impacto positivo o negativo generado a partir de ellas, pero siempre en consecuencia con su praxis coherente y liberadora.

- Y por último, teniendo en cuenta el carisma de este movimiento, el «teológico», 
estructurado a la luz de la fe y los «principia fidei» (la revelación), ya que esa fe ilumina la historia y la praxis en un «sentido crístico» que solo esta puede descubrir (Scanonne, 1982, p. 374). ${ }^{5}$

Se infiere cómo la vivencia de seres plurales como los cristianos «iluminados» permite hacer una exégesis dentro de la TL para fundamentar entre los sujetos una epistemología andante, hecha acción en una coherencia casi mágica con las prácticas de justicia en medio de la sociedad latinoamericana y como se afirma con Scannone en lo anterior, en ese sentido crístico que solo esa persona puede descubrir.

Pasando a otro momento de la búsqueda de los fundamentos de la TL, García Martínez (2010) narra que aquella epistemología se une en medio de la palabra revelada con la realidad, proponiendo que la TL busca ser una reflexión de la fe que en contacto con la palabra revelada ${ }^{6}$ y la realidad se enriquece en la acción transformadora, ya que la palabra ilumina y ayuda a interpretar la manifestación histórica de esta realidad. Teniendo en cuenta lo anterior, se evidencia que la TL representa un movimiento teológico plural, dado que, al implementar la categoría de liberación, le obliga a salir de los contextos bíblicos para darle su carácter de movimiento de acción frente a la realidad que le cobija (p. 289).

En este orden de reflexión, es donde se ubica un nexo con las ciencias sociales que abre

5| Es necesario aclarar que la TL presenta una faceta de «Jesucristo liberador», el cual se manifiesta dentro de las diversas escrituras como aquel líder político que enfrenta al Estado por ser corrupto y no darle al pueblo los servicios a los que tienen derecho. Para profundizar el término de «Jesucristo liberador» puede buscarse Leonardo Boff. Jesucristo el liberador. Editorial Sal Terrae.

6 | En su caso los escritos del éxodo en su exegesis sobre el proceso de liberación. pie para la segunda fase realizada dentro de la investigación.

Se resalta al final de la primera fase, que la $T L$ defiende la posición de la «dignidad humana» frente a cualquier situación de injusticia, y que esta ha sido el principal postulado epistemológico y su mensaje a la iglesia en el mundo, argumentado por Gustavo Gutiérrez (1975) en Teología de la Liberación: Perspectivas, donde se asume la TL como una «reflexión crítica de la praxis histórica» que se hace liberadora y también transformadora de la historia de la humanidad. Esta reflexión no se limita solo a pensar el mundo, sino que hace un llamado a cambiar el mundo injusto, luchando contra el despojo de esas mayorías oprimidas por las minorías, «en el amor que libera, en la construcción de una nueva sociedad, justa y fraternal» (p. 41).

\section{Fase integrativa: posibles aportes de la TL a la enseñanza de las ciencias sociales}

En consecuencia con la fase anterior y el objetivo que orientó la investigación: «contribuir a la enseñanza de las ciencias sociales en Colombia desde los postulados epistemológicos de la Teología de la Liberación», se resalta el fundamento metodológico implementado por la TL, el cual está denominado como «una aplicación contextualizada del método propio de la Teología de la Liberación: ver -mediación

7| La expresión 'iglesia' no debe entenderse desde la jerarquía propia del Vaticano, ni de una religión en específico (papa, obispo, arzobispo, sacerdote, seminarista, misionero, seglar), sino desde al origen de la palabra. Iglesia pro-

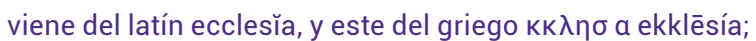
lo que es propiamente 'asamblea'. Entonces la iglesia es una comunidad de sujetos que se reúnen en un lugar determinado con un fin común. 
socioanalítica-; juzgar -mediación hermenéutica- y actuar -mediación práctica-» (García Martínez, 2010, p. 292). En este método, lo fundamental es que el sujeto comprenda su realidad histórica, social, económica y política, se reconozca como protagonista y responsable de su propia transformación consecuente con su praxis liberadora (Boff, 1975, p. 56).

Para hacer explícito el método «ver-juzgar-actuar» se toma como ejemplo el evangelio del camino de Emaús (Lc 24:13-35), en el que se evidencia la dinámica generada en términos de la TL. El «ver» representa el análisis de la realidad, el escuchar la realidad. El evangelio se encuentra entre los versículos 13-21. En este Jesús se aparece a dos discípulos que iban caminando hacia Emaús y les pregunta: «¿Qué pláticas son estas que tenéis entre vosotros mientras camináis, y por qué estáis tristes?» (Lc 24:17). Jesús indaga, observa, escucha a los discípulos para darse cuenta de qué es lo que sucede, aunque él mismo sabía qué era y buscaba que ellos también se percataran de su presencia. $Y$ tan sorprendidos estuvieron que le responden con otra pregunta: «¿Eres tú el único forastero en Jerusalén que no has sabido las cosas que en ella han acontecido en estos días?» (Lc 24:19). Jesús se hace el desentendido y dice: «¿Qué cosas?» (Lc 24:20), con el fin de continuar en el análisis del contexto, es decir, escuchar, afectarse, porque el ver incita a preguntar, con la limitación que se ve desde la posición personal. En el conocimiento de la realidad presente desde el ver, se observan aquellos fenómenos y funcionamiento de las estructuras sociales, las cuales emergen como componentes fundamentales a la hora de interpretar posibles causas del subdesarrollo y exclusión.

Aclarado el ver, se procede al juzgar, para ello se considera la ayuda de textos. En el ejemplo citado,
Jesús procede a usar las escrituras de su época. El evangelio relata qué les dijo a los caminantes.

¡Oh insensatos y tardos de corazón para creer todo lo que los profetas han dicho! ¿No era necesario que el Cristo padeciera estas cosas, y que entrara en su gloria? Y comenzando desde Moisés, y siguiendo por todos los profetas, les declaraba en todas las Escrituras lo que de él decían (Lc 24:25-27).

Juzgar, posterior a leer, tiene que ver con la ayuda de fuentes para medir e iluminar la realidad para entenderla mejor y así poder ser transformada. No solo basta con aceptar la realidad como es (injusta), hace falta tener criterios claros para juzgarla y a través de ello transformarla.

Finalmente, se considera el actuar, entendido como la «epistemología de la praxis». Esta epistemología «avala la interrelación mutua entre reflexión crítica e intervención social liberadora» (García Martínez, p. 292). Es decir, luego de analizar el contexto, se interviene generando una acción, una propuesta que pueda transformar la realidad. En el pasaje bíblico se entrega un actuar interpretativo y cambiante. Jesús es obligado a quedarse en casa de los discípulos (Lc 24:28) y este comienza a actuar de la misma manera como lo hacía en vida. «Sentado con ellos a la mesa, tomó el pan y lo bendijo, lo partió, y les dio. Entonces les fueron abiertos los ojos, y le reconocieron; mas él se desapareció de su vista.» (Lc 24:30-31). Luego de ello, los discípulos reaccionan y sienten la presencia actuante de Jesús, además, que debían ir donde los once discípulos, porque ellos se preguntaron «¿No ardía nuestro corazón en nosotros, mientras nos hablaba en el camino, y cuando nos abría las Escrituras?» (Lc 24:32). Y comprendido y asimilado en el gesto realizado por Jesús (ver-juzgar), ellos se levantaron y 
fueron a anunciar lo sucedido (actuar) porque habían sido transformados en este proceso; este encuentro en el que perciben la presencia de Jesús solo en el momento de partir el pan, acto memorable del Nazareno en vida en el que compartía con sus cercanos.

El anterior es grosso modo el proceso ver-juzgar-actuar, que analiza, entiende y muestra una acción al sujeto que transforma la realidad de una manera profunda, cueste lo que cueste si la realidad es injusta.

Ahora bien, desde un enfoque pedagógico, García Martínez (2010) retoma a Paulo Freire y a Enrique Dussel para resaltar el aspecto crítico y propositivo de la TL:

El discurso liberador conlleva una necesaria labor de concientización; desde esta perspectiva representa ser una toma de conciencia crítica de la realidad de la opresión y exclusión social. Se inicia una diacronía desde la negatividad histórica hasta la positividad dentro de un movimiento espiral progresivo. Ciertamente, el sujeto oprimido necesita de un tipo de racionalidad explicativa que pueda evidenciar la «cultura de la dominación» (García Martínez, 2010, p. 294).

Esta idea riñe con lo que algunos autores han denominado educación tradicional, y abre el camino a posteriores teorías latinoamericanas, especialmente desde Paulo Freire, quien entra al escenario con postulados como «pedagogía de la liberación» y Enrique Dussel con «ética de la liberación». En esta línea,

La Teología de la Liberación contiene intrínsecamente una labor pedagógica de naturaleza liberadora, ya que sin conciencia ética y crítica no existe posibilidad alguna de liberación. Siempre será en función de las víctimas de la historia, de los excluidos y de los discriminados, donde la ética encontrará su justificación y legitimidad últimas en la praxis liberadora (García Martínez, 2010, p. 294).

Finalmente, se evidencia una suerte de hermenéutica liberadora, a través de la «praxis histórica», la cual es transmutada en el referente de esperanza para aquellos que en el discurso son asumidos como oprimidos. Esa esperanza va a partir de un compromiso en donde dicho sujeto oprimido pueda convertirse en un sujeto histórico, protagonista de su «propio proceso de liberación» (García Martínez, 2010, p. 296).

\section{Resultados: Fase propositiva}

En los resultados se hace explícita la tercera fase del proceso. En primera instancia, según lo investigado y lo planteado por Scanonne en «el método de la Teología de la Liberación» se encuentra que, como resultado existe una ausencia de la $T L$ en la enseñanza de las ciencias sociales y, además, se encuentra un postulado epistemológico que se ha llamado «Epistemología de la libertad» que surge de los niveles epistemológicos de la TL mencionados anteriormente. Lo anterior es argumentado teniendo en cuenta que la $T L$ permite al sujeto en estos niveles construir su experiencia de fe a partir del conocimiento de la realidad, que permite al sujeto teológico hallar el camino para su construcción como sujeto crítico. En consonancia con la enseñanza de las ciencias sociales se argumenta, que dicha Epistemología de la libertad permite al docente un carácter pedagógico, didáctico y discursivo acorde con el contexto que se le presente y que brindando un análisis de la realidad en medio de 
la enseñanza a sus estudiantes, comenzará a construir una objetividad discursiva en medio de las subjetividades presentes.

Como segundo hallazgo aparece un concepto a partir del método de la TL que se puede denominar como «Epistemología de la realidad histórica», ya que la Teología de la Liberación obliga a salir de los contextos bíblicos para darle su carácter de movimiento de acción frente a la realidad que cobija al sujeto. En consonancia con un aporte a la enseñanza de las ciencias sociales, y en miras de la construcción de un discurso latinoamericano, suscita nuevamente a replantear los lineamientos curriculares propuestos por el MEN, para ser conscientes de la realidad histórica, en la que el continente y su discurso no contiene unos «latinoamericanismos» como lo plantea dicho documento, sino una construcción pedagógica, ética, teológica crítica de la realidad, que brinda al docente de ciencias sociales una mirada nueva a su contexto, y no solo la que exige el paradigma positivista de carácter procedimental y memorístico.

Finalmente, y como tercer hallazgo, se propone la necesidad de articular los lineamientos curriculares entregados por el MEN desde las epistemologías del Sur, construidas a partir del discurso crítico latinoamericano donde se incluyen las mencionadas anteriormente (Pedagogía de la Liberación, Ética de la liberación y esta, la Teología de Liberación), resaltando la crítica a las teorías de la dependencia. Eso sí, teniendo en cuenta que la enseñanza de las ciencias sociales podría verse altamente favorecida con los aportes del componente epistemológico desde el «ver, juzgar y actuar» al hacerlo parte de la estrategia metodológica. Ello posibilitará al docente de ciencias sociales estructurar un proceso de formación dentro y fuera del aula, en coherencia con la realidad del contexto.

\section{Discusión}

La TL propone numerosos aportes epistemológicos a la enseñanza de las ciencias sociales, ya que entiende que se debe enseñar en pro de la construcción del discurso latinoamericano para obtener un verdadero desarrollo. Pero ¿qué es lo que imposibilita que esta epistemología haga parte del discurso institucional?

La TL, como otras epistemologías del Sur que hoy día aparecen desde la educación popular en Colombia, ha buscado la manera de ingresar al carácter institucional de la educación para la construcción del discurso latinoamericano en Colombia y se conecta con el objetivo de la enseñanza de las ciencias sociales, como lo afirma Cajiao argumentando:

El objetivo de la enseñanza de las ciencias sociales es lograr que la persona sea capaz de hacer una reflexión ordenada y comprensiva acerca de su acontecer individual, inmerso y condicionado por un entorno social cuya realidad actual es el resultado de un proceso histórico, a lo largo del cual los grupos humanos han construido formas de organización social, formas de relación con el espacio físico, formas características de expresión, formas de producción, intercambio y distribución de la riqueza, formas particulares de interpretar la realidad... Este proceso de reflexión, a su vez, tiene sentido en cuanto permita al individuo, por la vía del entendimiento racional, intervenir como persona y como colectividad en la modificación de las condiciones de vida heredadas culturalmente, con el fin de ser protagonista activo y responsable en la construcción de nuevos modelos de sociedad y cultura (Cajiao, 1999 p. 18). 
Pero al estructurar unos lineamientos en medio de un paradigma que es traído de otro continente, las posibilidades de ingresar se limitan.

Así mismo, los lineamientos curriculares mencionan el concepto de «latinoamericanismo», es decir, reducen cada epistemología propia a lo que Santiago Castro y Eduardo Mendieta llaman al discurso latinoamericano como «Teorías sin disciplina» (MEN, 2002, p. 26), llevando las epistemologías del Sur a una categoría que deja sin criterio las construcciones propias del contexto.

Si bien es cierto que estas alternativas de pensamiento son recientes y posiblemente estén descontextualizadas según los críticos, es precisamente porque el sistema educativo colombiano está apuntando hacia dinámicas que generan procesos que alimentan la idea de progreso impuesta desde afuera.

La TL dista de la injusticia e inequidad patrocinadas desde las políticas neoliberales. Leonardo Boff dice (1975) que la centralidad de dicha corriente es generar «un hombre nuevo y una forma más humana de sociedad por medio de una praxis consecuente y liberadora» (p. 56). Y esa praxis consecuente conduce a la sociedad a plantearse que la educación y, sobre todo las ciencias sociales, deben proyectar al país hacia la equidad, enseñando que no siempre pueden ser los mismos con las mismas, como alguna vez dijo Jaime Bateman, ex comandante de la guerrilla del M-19. La Teología de la Liberación llama a una conciencia de clase, la cual fue promulgada por Camilo Torres; y la misma que hoy día sigue vigente considerando, además, la unión en lo diverso, para la construcción de un país más justo.
Este artículo, que encuentra ausente la TL en la enseñanza de las ciencias sociales, ofrece en la línea de la construcción «del sur» una alternativa desde la perspectiva crítica latinoamericana como su mayor aporte a los procesos educativos, posicionando al docente dentro del aula de clase como un guía que propicia experiencias pedagógicas como una opción liberadora; todo lo escrito aquí es parte de lo que la TL y las demás epistemologías del Sur han promulgado, aun siendo recientes y teniendo largo camino por recorrer en lo que viene de la historia para demostrar a los detractores que no son ninguna teoría sin disciplina.

Para cerrar, se propone sobre aquello que dijo Modesto Guerrero en el texto homenaje a los 48 años de la muerte del Cura y Sociólogo Camilo Torres Restrepo:

Como todo mundo lo sabe, en cada época de la historia se ha adoptado un adjetivo para colocar fuera de la ley a los individuos. Me parece que así como en la primera época de nuestra era se decía cristiano ${ }^{8}$ a una persona que se quería poner en contra de la ley, aunque no fuera cristiano, hoy en día se usa el adjetivo comunista para poner fuera de ley a aquellos inconformes (Guerrero, 2014, p. 351).

El poder tiene miedo al cambio y la TL busca cambiar estructuras. Por tanto, queda a criterio del lector resolver si ¿La TL y las nuevas epistemologías son ahora el comunismo o cristianismo del siglo XXI?... La discusión, queda abierta.

8| Y esto demuestra, entonces, históricamente porque la TL como movimiento social asume esa defensa de la dignidad humana en medio del cristianismo según la vocación de sus fundadores. 


\section{Referencias}

Boff, L. (1975). Teología del cautiverio y la liberación. Bogotá: Paulinas.

Cajiao, F. (1999). Pedagogía de las ciencias sociales. Bogotá: Tercer Mundo.

Domingo Moratalla, T. (1 de enero de 2001). UNED. Obtenido de UNED: http://e-spacio.uned.es/fez/ view/bibliuned:InvFen-2001-3-5180

Flórez Ochoa, R. (1994). Hacia una pedagogía del conocimiento. Bogotá: McGraw-Hill.

García Martínez, J. (2010). La epistemología de la teología de la liberación y su hermenéutica desde la praxis. TELOS, 12(3) 287-297.

Guerrero, M. E. (2014). Camilo y el camino andado. En V. Autores, Unidad en la diversidad: Camilo Torres y el Frente Unido del pueblo (Aportes para el debate) (p. 396). Bogotá: Desde Abajo.

Gutiérrez, G. (1975). Teología de la liberación: Perspectivas. Salamanca: Sígueme.

Latina, S. B. (1960). La biblia. Valera.

Scanonne, J. C. (10 de septiembre de 1982). El método de la teología de la liberación. Obtenido de Theologica Xaveriana. http://theologicaxaveriana.javeriana.edu.co/descargas. php?archivo=Juan\%20Carlos.pdf\&idArt=845\&edicion=73

\section{Lecturas sugeridas adicionales}

Boff, L. (7 de abril de 2014).«La Iglesia de los pobres en el siglo XXI» https://www.youtube.com/ watch?v=IPLEjVJx6-4

Boff, L. (1998). Iglesia, Carisma y Poder. España: Sal Terrae.

Flórez López, J. A. (2007-2008). Diálogo Interreligioso: Cristianismo y Pueblos Indígenas. Anuario FUCLA-FUNDACIÓN UNIVERSITARIA CLARETIANA, 269-280.

Flórez Ochoa, R. (1994). Hacia una pedagogía del conocimiento. Bogotá: McGraw-Hill.

MEN. (2002). Lineamientos Curriculares de ciencias sociales. Bogotá: MEN. Obtenido de http://www. mineducacion.gov.co/1621/article-87874.html

MEN. (2003). Estándares Básicos de competencia. Bogotá: MEN. Obtenido de http://www.mineducacion. gov.co/1621/articles-116042_archivo_pdf3.pdf

Pablo VI. (2011). Populorum Progressio. Bogotá: San Pablo.

Rodríguez Albarracín, E. (1992). Teología de la liberación Debate y perspectivas. Bogotá: Antropos.

Scannone, J. C. (1982). La teología de la liberación. Caracterización, corrientes, etapas. Stromata.

Wallerstein, I. (2003). Impensar las ciencias sociales. México: Siglo XXI.

Wallerstein, I. (2007). Abrir las ciencias sociales. México: Siglo XXI. 